\title{
Adaptive Control Design with S-Variable LMI Approach for Robustness and $L_{2}$ Performance *
}

\author{
Dimitri Peaucelle and Harmony Leduc \\ LAAS-CNRS, Univ. Toulouse, CNRS, Toulouse, France
}

December 19, 2018

\begin{abstract}
Matrix inequality based results are proposed for the design of direct adaptive control. The focus is on guaranteeing robustness of the closed-loop when facing both structured parametric uncertainties and input disturbances. Results apply to systems in descriptor form with polytopic uncertainties and readily extend to systems rational in the uncertain parameters. The exposed methodology is an extension of previous studies started in cooperation with Alexander L. Fradkov. These studies aim at applying up-to-date linear robust control tools for passivity-based adaptive control. The paper contributes to this framework with new parameter-dependent Lyapunov functions and new degrees of freedom in the adaptive laws offering the possibility to design controllers with improved closed-loop performances.

Keywords: Adaptive control; Uncertain polytopic systems; Descriptor systems; LMIs; Practical stability; $L_{2}$ performance.
\end{abstract}

\section{Introduction}

Adaptive control is an attractive methodology to improve performances and robustness of closed-loop controlled systems in comparison to linear time-invariant control. Its potentials have been studied for decades starting from intuitive gradient adaptation and then growing in maturity with theoretical Lyapunov-based results such as in [26]. Adaptive control strategies share the idea of adjusting with non-linear rules the parameters of the control law, but have various more or less complex architectures. In [2] the central viewpoint is to adjust parameters of a classical two-degrees of freedom controller to bring the closed-loop to behave as a given reference model. It leads to controllers containing the reference model, several filters and the adaptive two-degrees of freedom controller which is close to be of the same order of the plant. Results are essentially for linear single-input single-output systems, but extensions are also provided for non-linear systems using back-stepping strategies. Such results are extended to more general non-linear systems in [21] in which the filters are explicitly described as elements for parameter-estimation, and combined to the control strategy. These methods are shown to be related to speed-gradient approches in [15]. Such combined estimation/control enters the classification of [18] as indirect adaptive strategies which require estimation of parameters. More recent results such as [16] increase further the complexity of the adaptation rule with estimates of the states of the plant, even when plant states are actually measured. In [1] adaptation is revisited with a new methodology based on immersion and invariance which can be seen as an extension of stable attractive sub-manifolds discussed in [15]. The exposed adaptive strategy leads to output feedback adaptive controllers that include estimators of both the state and the unknown parameters, and requires, as in model reference adaptive control, the a priori definition of ideal dynamics that drive the adaptation rule. Opposite to this indirect adaptive control strategy, the direct (or simple) adaptive control described in [20] applies the adaptation rules directly to the controller parameters without necessarily augmenting the controller architecture with filters for estimation and observation.

In results discussed above, improvements are often claimed in terms of robustness. This claim is reasonable for indirect adaptive controllers that construct and use estimates of the uncertainties, but it is at the expense of high order controllers and complex intricate laws. Moreover, it sometimes requires restrictive assumptions such as "matching conditions" meaning that uncertainties and control inputs are in the same

* The Version of Record of this manuscript has been published and is available in International Journal of Control 2018 https://www.tandfonline.com/doi/abs/10.1080/00207179.2018.1554907 
subspace. Proving robustness is less trivial for direct adaptive control. It often needs strong assumptions such as passivity in $[12,32]$ or almost passivity in $[14,4]$.

To go beyond these restrictive assumptions, to keep the adaptive law as simple as possible, and to provide constructive design methods, we have started together with Alexander L. Fradkov to introduce tools used in linear robust control for the direct adaptive control design. Preliminary work includes [27] in which the passivity assumptions are kept but handled with linear matrix inequalities (LMIs), or [28] for first results concerning $L_{2}$ performance in the robust-adaptive context. These results were further extended and applied to satellite examples in [29]. Alternative related robust-adaptive results are in [8] where LMI methods are used to design parameter-dependent state-feedback controllers in which the parameters are estimates obtained by an adaptive law, and in [35] where LMI methods are proposed for the analysis of strict positive realness of closed-loop systems with simple adaptive control, results extended to the discrete-time case in [6], and which consider $H_{\infty}$ performance in [5]. At the difference of our results these last cited contributions do not address the design of the adaptive controllers.

Within this robust-adaptive framework, the goal of the present paper is to design a simple adaptive controller which ensures that the closed-loop system is stable, and to minimize an input-output performance (robustness to disturbances), while guaranteeing these properties whatever uncertainties on the model parameters bounded in given intervals (robustness to parametric uncertainties). The employed tools are polytopic modeling of uncertain systems (as in upper cited references in the robust-adaptive contexts, but at the difference of [22] where uncertainties are norm-bounded), Lyapunov theory, and results are formulated in terms of matrix inequalities $([7])$. Thanks to recent results from $[34,25]$ the polytopic representation is not restrictive for systems rationally dependent on the uncertain parameters, but this is at the expense of reformulating the system in descriptor form where the dynamics are driven by an implicit linear application. Results are formulated in terms of matrix inequalities and rely on quadratic parameter dependent Lyapunov functions. The ability to handle descriptor systems and to introduce parameter-dependent Lyapunov functions is thanks to the S-variables approach described in details in [11], approach which is inspired of the S-procedure by [36].

Results of this paper are extensions of [23] and are applicable to any linear uncertain system for which one has designed a stabilizing linear time-invariant (LTI) control. At the difference of results such as [6] no passivity property is needed, at least not explicitly (asymptotically stable linear systems can be seen as passive for properly chosen inputs/outputs by eventually adding a parallel feedforward gain as stated in $[3,19,13])$. The initial LTI control for the given plant is converted using LFTs $([10])$ into an equivalent problem of diagonal static output feedback control for an augmented plant. Adaptation is applied to tune in real time the gains of this diagonal control. The adaptive rule involves a weighted sum of the measured signals from the augmented plant and a projection function as in [30] which guarantees bounded adaptive gains. The crucial design problem is to tune the weighted sum and the set in which the adaptive gains are constrained to be. Compared to [28] the results we propose have the following improvement: they apply to descriptor systems and hence to any linear system rationally dependent of uncertain parameters. Compared to [23] the improvements are: to consider $L_{2}$ performance; to build Lyapunov functions in which all terms are parameter-dependent; and that the adaptive gains evolve in sets which are not centered at the initially given LTI control gain. This last feature allows to improve significantly the performance but is at the expense of non-convexity in the design of the adaptive law parameters. The design is based on a heuristic iterative LMI scheme inspired from existing ones for static-output feedback design (see [31] for a survey about these heuristics).

The outline of the paper is as follows. First in preliminaries we recall the framework of descriptor systems with polytopic uncertainties and the LMI results that allow to compute upper bounds on the robust $L_{2}$ performance of these LTI systems. Then Section 3 exposes the main results of the paper, namely the matrix inequalities for the analysis of the performance of the proposed adaptive control, its implications in terms of Lyapunov inequalities and the heuristic algorithm for the design of the parameters. Section 4 is devoted to an illustrative numerical example. Section 5 draws some conclusions and is followed by an Appendix which contains the proofs of the main results.

Notation: $I$ stands for the identity matrix. $A^{T}$ is the transpose of the matrix $A .\{A\}^{\mathcal{S}}$ stands for the symmetric matrix $\{A\}^{\mathcal{S}}=A+A^{T}$. $\operatorname{Tr}(A)$ is the trace of $A$. If $f$ is a vector $F=\operatorname{diag}(f)$ stands for the diagonal matrix whose diagonal elements are the coefficients $f_{i}$ of $f$. For a matrix $A \in \mathbb{R}^{n \times m}$ of $\operatorname{rank} r$, $A^{\perp} \in \mathbb{R}^{(n-r) \times n}$ stands for the matrix of maximal rank such that $A^{\perp} A=0$, and $A^{\circ} \in \mathbb{R}^{m \times r}$ stands for the full rank matrix such that $A A^{\circ}$ is full rank. $A \succ B$ is the matrix inequality stating that $A-B$ is symmetric positive definite. The terminology "congruence operation of $A$ on $B$ " is used to denote $A^{T} B A$. If $A$ is full column rank, and $B \succ 0$, the congruence operation of $A$ on $B$ gives a positive definite matrix: $A^{T} B A \succ 0$. 
A matrix inequality of the type $N(X) \succ 0$ is said to be a linear matrix inequality (LMI for short), if $N(X)$ is affine in the decision variables $X . \Xi_{\bar{v}}=\left\{\xi_{v=1 \ldots \bar{v}} \geq 0, \sum_{v=1}^{\bar{v}} \xi_{v}=1\right\}$ is the unitary simplex in $\mathbb{R}^{\bar{v}}$. The coefficients $\xi_{v}$ of $\xi$ are barycentric coordinates of uncertain polytopic matrices. Throughout this paper uncertainties are assumed constant $(\dot{\xi}=0)$.

\section{Preliminaries}

\subsection{Descriptor systems with uncertainties}

Let us consider linear systems in descriptor form with dynamics represented by implicit differential equations as follows

$$
E_{x}(\xi) \dot{x}(t)+E_{\pi}(\xi) \pi(t)=A(\xi) x(t)+B_{w}(\xi) w(t)+B_{u}(\xi) u(t)
$$

where $x(t) \in \mathbb{R}^{n_{x}}$ is the state, $w(t) \in \mathbb{R}^{m_{w}}$ is a perturbation, $u(t) \in \mathbb{R}^{m_{u}}$ is the control input and $\pi(t) \in \mathbb{R}^{n_{\pi}}$ is an auxiliary signal algebraically linked to the others via the $n$ scalar equations of the matrix representation

$$
M(\xi)=\left[\begin{array}{lllll}
E_{x}(\xi) & E_{\pi}(\xi) & -A(\xi) & -B_{w}(\xi) & -B_{u}(\xi)
\end{array}\right] \in \mathbb{R}^{n \times\left(n_{x}+n_{\pi}+n_{x}+m_{w}+m_{u}\right)}
$$

A special case of such representation is the classical state-space representation

$$
\dot{x}(t)=A(\xi) x(t)+B_{w}(\xi) w(t)+B_{u}(\xi) u(t)
$$

which corresponds to the situation when $n_{\pi}=0, n=n_{x}$ and $E_{x}(\xi)=I_{n}$.

The advantage of the descriptor representation involving differential-algebraic equations is that it includes a more general class of systems (see [17] for a complete overview) and it is a more natural way of representing systems when building models based on physical laws and when subsystems have complex interconnections. The auxiliary signal $\pi$ is used as an alternative to other more classical descriptor representations where signals such as $\pi$ are included in the 'state' $x$ and where the $E_{x}$ matrix is assumed square. The advantages of our formulation, borrowed from [9], is that it allows to concentrate on the true states of the system, and to bring new freedom for modeling. One of such degrees of freedom, is to transform any system rational in the uncertainties into a model in which the system matrices are affine in the uncertainties (see [11]). For this reason we shall assume without loss of generality compared to the case when matrices are rational in the uncertain parameters, that $M(\xi)$ is a polytopic matrix described by

$$
M(\xi)=\sum_{v=1}^{\bar{v}} \xi_{v} M^{[v]} \quad: \quad M^{[v]}=\left[\begin{array}{lllll}
E_{x}^{[v]} & E_{\pi}^{[v]} & -A^{[v]} & -B_{w}^{[v]} & -B_{u}^{[v]}
\end{array}\right]
$$

where $\bar{v}$ is the number of vertices of the polytope, a generic vertex being denoted $M^{[v]}$, and the barycentric coordinates $\xi_{1}, \ldots, \xi_{\bar{v}}$ belong to the simplex $\Xi_{\bar{v}}$.

Assumption 1 It is assumed that one can build a factorisation $\left[\begin{array}{cc}E_{x}(\xi) & E_{\pi}(\xi)\end{array}\right]=E_{1}(\xi)\left[\begin{array}{ll}E_{2 x} & E_{2 \pi}\end{array}\right]$ such that $E_{1}(\xi)=\sum_{v=1}^{\bar{v}} \xi_{v} E_{1}^{[v]} \in \mathbb{R}^{n \times q}$ is full column rank for all uncertainties $\xi \in \Xi_{\bar{v}}$, and where $E_{2 x} \in$ $\mathbb{R}^{q \times n_{x}}$ and $E_{2 \pi} \in \mathbb{R}^{q \times n_{\pi}}$. Let $r_{\pi} \leq n_{\pi}$ be the rank of $E_{2 \pi}$, define $E_{2}=E_{2 \pi}^{\perp} E_{2 x} \in \mathbb{R}^{\left(q-r_{\pi}\right) \times n_{x}}$, and let $r_{x} \leq n_{x}$ be the rank of $E_{2}$.

General descriptor systems as defined by equation (1) have two special features in comparison to statespace representations (3):

- Algebraic constraints may impose linear dependence between components of the state (and of the auxiliary signal $\pi$ ). The equilibrium whose (asymptotic) stability shall be studied is hence not a single point but the null space of a linear application. More precisely, we shall study the stability of the $n_{x}-r_{x}$ dimensional sub-space $E_{2} x=0$.

- Initial conditions on the state $x(0)$ may not satisfy the algebraic constraints. In such case the differential equations impose that these states shall jump to some other value (reset step type function on the states) at time $t=0^{+}$. This corresponds to impulses on the derivatives of the states and is a common feature of descriptor systems. Due to the algebraic equations it is possible to build representations of the type (1) in which the impulses of the derivatives imply also impulses on some components of the state. The systems considered in this paper shall not have such impulses on the states and are said impulse-free. This assumption is common to all LMI-based results for descriptor systems. 
Assumption 1 allows the derivation of results for descriptor systems with uncertainties in the $E_{x}$ and $E_{\pi}$ matrices which is usually not the case in the litterature. But it imposes that the equilibrium subspace $E_{2} x=0$ and the impulse-free nature of the system are both independent of the uncertainties.

\subsection{Diagonal static output-feedback control}

We shall consider output-feedback control $u=K_{o} y$ and performances in terms of $L_{2}$ induced norm of the transfer from the disturbance $w$ to an exogenous output $z$. Both equations defining $y(t) \in \mathbb{R}^{p}$ and $z(t) \in \mathbb{R}^{p z}$ are for simplicity assumed to be uncertainty independent:

$$
z(t)=C_{z} x(t)+D_{z w} w(t) \quad, \quad y(t)=C_{y} x(t)
$$

Assumption 2 Without loss of generality we assume that the system is square, $p=m_{u}$ and that $K_{o}=$ $\operatorname{diag}\left(k_{o}\right)$ is a diagonal matrix.

See Appendix A for the proof that the assumption is lossless. Any dynamic LTI control may be equivalently converted to this case.

\subsection{Robust $L_{2}$ performance of descriptor systems}

Assumption 3 It is assumed that one has pre-designed a diagonal static output-feedback control $u=K_{o} y$, $K_{o}=\operatorname{diag}\left(k_{o}\right) \in \mathbb{R}^{p \times p}$ such that there exist $\hat{P}^{[v]}=\hat{P}^{[v] T} \in \mathbb{R}^{\left(q-r_{\pi}\right) \times\left(q-r_{\pi}\right)}, \hat{Y}^{[v]} \in \mathbb{R}^{n_{x} \times\left(n_{x}-r_{x}\right)}, \hat{S} \in$ $\mathbb{R}^{\left(q+n_{x}+m_{w}\right) \times n}, \hat{\epsilon} \geq 0$ and $\gamma_{o}^{2}$ solution to the following LMIs for all $v=1 \ldots \bar{v}$ :

$$
\begin{gathered}
\left(E_{2} E_{2}^{\circ}\right)^{T} \hat{P}^{[v]}\left(E_{2} E_{2}^{\circ}\right) \succ 0 \\
\left\{\hat{N}_{2 x}^{T} \hat{P}_{e}^{[v]} \hat{N}_{1 x}+\hat{S} \hat{M}_{c}^{[v]}\right\}^{\mathcal{S}}+\hat{\epsilon} \hat{N}_{2 x}^{T} E_{2}^{T} E_{2} \hat{N}_{2 x}+\hat{N}_{z}^{T} \hat{N}_{z}-\gamma_{o}^{2} \hat{N}_{w}^{T} \hat{N}_{w} \prec 0
\end{gathered}
$$

where

$$
\begin{gathered}
\hat{N}_{1 x}=\left[\begin{array}{lll}
I_{q} & 0_{q, n_{x}} & 0_{q, m_{w}}
\end{array}\right], \quad \hat{N}_{2 x}=\left[\begin{array}{lll}
0_{n_{x}, q} & I_{n_{x}} & 0_{n_{x}, m_{w}}
\end{array}\right], \\
\hat{N}_{z}=\left[\begin{array}{lll}
0_{p_{z}, q} & C_{z} & D_{z w}
\end{array}\right], \quad \hat{N}_{w}=\left[\begin{array}{lll}
0_{m_{w}, q} & 0_{m_{w}, n_{x}} & I_{m_{w}}
\end{array}\right], \\
\hat{M}_{c}^{[v]}=\left[\begin{array}{lll}
E_{1}^{[v]}-\left(A^{[v]}+B_{u}^{[v]} K_{o} C_{y}\right) & -B_{w}^{[v]}
\end{array}\right], \\
\hat{P}_{e}^{[v]}=\left(E_{2}^{T} \hat{P}^{[v]}+\hat{Y}^{[v]} E_{2}^{\perp}\right) E_{2 x \pi}^{\perp} .
\end{gathered}
$$

According to [11] Assumption 3 implies that the closed-loop with static output-feedback gain $u=K_{o} y$ is robustly stable and $\gamma_{o}$ is a guaranteed upper-bound on the robust $L_{2}$ induced norm of the closed-loop system. We do not give all the details of the proof of this result here (see [11]) but in order to better understand the main adaptive control results that follow, let us give some key elements of the proof:

- Equation (6) allows to define a parameter-dependent Lyapunov function $V(x, \xi)=x^{T} E_{2}^{T} \hat{P}(\xi) E_{2} x$ where $\hat{P}(\xi)=\sum_{v=1}^{\bar{v}} \xi_{v} \hat{P}^{[v]}$ which is strictly positive for all $x$ such that $E_{2} x \neq 0$.

- Equation (7) allows to prove that whatever $\xi \in \Xi_{\bar{v}}$ the following property holds along the trajectories of the system $(1)(5)$ :

$$
\dot{V}(x, \xi)+\hat{\epsilon} x^{T} E_{2}^{T} E_{2} x+\left(z^{T} z-\gamma_{o}^{2} w^{T} w\right)<0
$$

In case of zero disturbances $(w \equiv 0)$ this implies that the derivative of the Lyapunov function is negative and the state converges asymptotically to the set $E_{2} x=0$. In case of zero initial conditions $E_{2} x(0)=0$, taking the integral over time of the inequality (8) implies $\|z\| \leq \gamma_{o}\|w\|$, thus proving the $L_{2}$ induced norm performance. 


\section{Main result}

\subsection{Diagonal direct adaptive control}

The goal of this paper is to design an adaptive control law based on the knowledge of $K_{o}$ for which we may prove improvements in terms of robust $L_{2}$ performance. The considered adaptive control is of direct adaptive type and defined for each $i=1 \ldots p$ by the following scalar equations:

$$
\begin{gathered}
u_{i}(t)=k_{i}(t) y_{i}(t), \\
\dot{k}_{i}(t)=\operatorname{proj}_{\mathcal{E}\left(k_{i c}, r_{i}\right)}\left(k_{i}(t), h_{i}(t)\right), \\
h_{i}(t)=-g_{i} G_{i} y(t) y_{i}(t)-\sigma_{i}\left(k_{i}(t)-k_{o i}\right)
\end{gathered}
$$

where $k_{i c} \in \mathbb{R}, r_{i} \in \mathbb{R}, G_{i} \in \mathbb{R}^{1 \times p}, g_{i} \in \mathbb{R}$ and $\sigma_{i} \in \mathbb{R}$ are parameters to be determined. Before getting to the main results, let us describe these adaptation rules.

- (9) indicates that the adaptive control mimics the static output-feedback control where the static gains $k_{o i}$ are replaced by dynamic gains. This structure is convenient in practice because it does not imply any modification in the control architecture compared to the initial LTI control. The compact version of (9) is denoted

$$
u(t)=K(t) y(t)
$$

with $K(t)=\operatorname{diag}(k(t)) \in \mathbb{R}^{p \times p}$.

- The proj function in (10) is built accordingly to [30] in such a way that the gains $k_{i}(t)$ are bounded in predefined sets $\mathcal{E}\left(k_{i c}, r_{i}\right)$. These predefined sets are intervals:

$$
k_{i} \in \mathcal{E}\left(k_{i c}, r_{i}\right) \quad \Leftrightarrow \quad\left(k_{i c}-k_{i}\right)^{2} \leq r_{i}^{2} \quad \Leftrightarrow \quad k_{i} \in\left[\begin{array}{ll}
k_{i c}-r_{i} & k_{i c}+r_{i}
\end{array}\right]
$$

where $k_{i c}$ is the center of the interval, $r_{i}$ is its radius. The larger is the scalar $r_{i}$, the wider is the interval. The $\operatorname{proj}_{\mathcal{E}\left(k_{i c}, r_{i}\right)}\left(k_{i}(t), h_{i}(t)\right)$ function works as follows:

- If $k_{i}(t)$ is in the interior of the set $\mathcal{E}\left(k_{i c}, d_{i}\right)$ then $\dot{k}_{i}(t)=h_{i}(t)$. The adaptation rule (11) is applied without any modification.

- If $k_{i}(t)=k_{i c}+r_{i}$ and $h_{i}(t) \geq 0$ then $\dot{k}_{i}(t)=0$. As soon as $h_{i}(t)<0$ then $\dot{k}_{i}(t)=h_{i}(t)$.

- If $k_{i}(t)=k_{i c}-r_{i}$ and $h_{i}(t) \leq 0$ then $\dot{k}_{i}(t)=0$. As soon as $h_{i}(t)>0$ then $\dot{k}_{i}(t)=h_{i}(t)$.

The differential equation (10) hence works as a saturated integrator with values saturated between $k_{i c}-r_{i}$ and $k_{i c}+r_{i}$. The result of the $\operatorname{proj}_{\mathcal{E}\left(k_{i c}, r_{i}\right)}\left(k_{i}(t), h_{i}(t)\right)$ function can also be understood as $\dot{k}_{i}(t)=h_{i}(t)+q_{i}(t)$ where most of the time $q_{i}(t)=0$. The situations when it is non-zero are

- $k_{i}(t)=k_{i c}+r_{i}$ and $h_{i}(t) \geq 0$. In such case $q_{i}(t)=-h_{i}(t) \leq 0$ and whatever $f_{i} \in\left[k_{i c}-r_{i}, k_{i c}+r_{i}\right]$ one has $\left(k_{i}(t)-f_{i}\right) q_{i}=\left(k_{i c}+r_{i}-f_{i}\right) q_{i} \leq 0$.

$-k_{i}(t)=k_{i c}-r_{i}$ and $h_{i}(t) \leq 0$. In such case $q_{i}(t)=-h_{i}(t) \geq 0$ and whatever $f_{i} \in\left[k_{i c}-r_{i}, k_{i c}+r_{i}\right]$ one has $\left(k_{i}(t)-f_{i}\right) q_{i}=\left(k_{i c}-r_{i}-f_{i}\right) q_{i} \leq 0$.

This allows to conclude that at all times $\dot{k}_{i}(t)=h_{i}(t)+q_{i}(t)$ with $\left(k_{i}(t)-f_{i}\right) q_{i} \leq 0$ whatever $f_{i} \in$ $\left[k_{i c}-r_{i}, k_{i c}+r_{i}\right]$.

For the ease of notations we shall denote $K_{c}, R, H(t), Q(t)$ diagonal $\mathbb{R}^{p \times p}$ matrices with components $k_{i c}, r_{i}{ }^{2}, h_{i}(t)$ and $q_{i}(t)$ on their respective diagonals, and the compact version of (10) is denoted

$$
\dot{K}(t)=\operatorname{proj}_{\mathcal{E}\left(K_{c}, R\right)}(K(t), H(t))=H(t)+Q(t)
$$

It works as a diagonal element-wise saturated integrator. Its properties are that (if all gains are initialized in the intervals) at all times one has $\left(K_{c}-K(t)\right)^{T}\left(K_{c}-K(t)\right) \leq R^{2}$ and $(K(t)-F) Q(t) \leq 0$ is element-wise negative whatever value $F \in \mathcal{E}\left(K_{c}, R\right)$. 
- The adaptation rule (11) contains two terms. The term $\sigma_{i}\left(k_{i}(t)-k_{o i}\right)$ with a positive value of $\sigma_{i}$ is a damping term that drives asymptotically the gain $k_{i}(t)$ back to $k_{o i}$ if the system has reached $y_{i} \equiv 0$. The term $g_{i} G_{i} y(t) y_{i}(t)$ pushes the gain $k_{i}(t)$ in a direction that depends of the product between $y_{i}(t)$ and a linear combination of all measured outputs $G_{i} y(t)$. The positive scalar $g_{i}$ tunes the speed of adaptation. In the following $\Gamma$ and $\Sigma$ are diagonal $\mathbb{R}^{p \times p}$ matrices with elements $g_{i}$ and $\sigma_{i}$ respectively on their diagonals, $G \in \mathbb{R}^{p \times p}$ is the matrix whose rows are the row vectors $G_{i} \in \mathbb{R}^{p \times p}$, and

$$
H(t)=-\Gamma \in G y(t) y^{T}(t)-\Sigma\left(K(t)-K_{o}\right)
$$

is the matrix version of the adaptation rule ( $\cdot$ denotes the element-wise product).

The proposed adaptive rule fits to the (diagonal) structure of the original controller $K_{o}$. This feature is useful for implementation as it was done in [29] because it does not require to change the control architecture when moving from LTI to adaptive control.

\subsection{Linear matrix inequalities for adaptive control}

We now state the main result of this paper. It involves the following matrix inequalities

$$
\begin{gathered}
{\left[\begin{array}{cc}
R^{2} & K_{c}-F^{[v]} \\
K_{c}-F^{[v]} & I
\end{array}\right] \succeq 0,} \\
{\left[\begin{array}{cc}
R^{2} & K_{c}-K_{o} \\
K_{c}-K_{o} & I
\end{array}\right] \succeq 0,} \\
\left(E_{2} E_{2}^{\circ}\right)^{T} P^{[v]}\left(E_{2} E_{2}^{\circ}\right) \succ 0, \\
\left\{N_{2 x}^{T} P_{e}^{[v]} N_{1 x}+S M_{c}^{[v]}\right\}^{\mathcal{S}}+\epsilon N_{2 x}^{T} E_{2}^{T} E_{2} N_{2 x}+\alpha\left(N_{z}^{T} N_{z}-\gamma^{2} N_{w}^{T} N_{w}\right) \\
+2 N_{y}^{T} R^{2} N_{y}-2 N_{y u}^{T} N_{K}^{T} N_{K} N_{y u}+\left\{N_{y}^{T} G^{T} N_{F}^{[v]} N_{y u}\right\}^{\mathcal{S}} \prec 0,
\end{gathered}
$$

where the notations are as follows

$$
\begin{gathered}
N_{1 x}=\left[\begin{array}{llll}
I_{q} & 0_{q, n_{x}} & 0_{q, m_{w}} & 0_{q, p}
\end{array}\right], \quad N_{2 x}=\left[\begin{array}{llll}
0_{n_{x}, q} & I_{n_{x}} & 0_{n_{x}, m_{w}} & 0_{n_{x}, p}
\end{array}\right], \\
N_{z}=\left[\begin{array}{lllll}
0_{p_{z}, q} & C_{z} & D_{z w} & 0_{p_{z}, p}
\end{array}\right], \quad N_{w}=\left[\begin{array}{llll}
0_{m_{w}, q} & 0_{m_{w}, n_{x}} & I_{m_{w}} & 0_{m_{w}, p}
\end{array}\right], \\
N_{y}=\left[\begin{array}{lllll}
0_{p, q} & C_{y} & 0_{p, m_{w}} & 0_{p, p}
\end{array}\right], \quad N_{y u}=\left[\begin{array}{cccc}
0_{p, q} & C_{y} & 0_{p, m_{w}} & 0_{p, p} \\
0_{p, q} & 0_{p, p} & 0_{p, m_{w}} & -I_{p}
\end{array}\right], \\
M_{c}^{[v]}=\left[\begin{array}{llll}
E_{1}^{[v]}-\left(A^{[v]}+B_{u}^{[v]} K_{o} C_{y}\right) & -B_{w}^{[v]} & -B_{u}^{[v]}
\end{array}\right], \\
N_{K}=\left[\begin{array}{ll}
K_{c}-K_{o} & I_{p}
\end{array}\right], \quad N_{F}^{[v]}=\left[\begin{array}{ll}
F^{[v]}-K_{o} & I_{p}
\end{array}\right], \\
P_{e}^{[v]}=\left(E_{2}^{T} P^{[v]}+Y^{[v]} E_{2}^{\perp}\right) E_{2 x \pi}^{\perp} .
\end{gathered}
$$

Theorem 1 Consider the system described by (1), (4), (5) and a static control gain $K_{o}$. The upper defined inequalities are such that:

(a) If conditions of Assumption 3 hold, then the constraints (16), (17), (18), (19) are feasible for all vertices $v=1 \ldots \bar{v}$ with the following choice of decision variables:

$$
\begin{gathered}
P^{[v]}=\alpha \hat{P}^{[v]}, \quad Y^{[v]}=\alpha \hat{Y}^{[v]}, \quad S=\left[\begin{array}{c}
\alpha \hat{S} \\
0
\end{array}\right], \quad \epsilon=\alpha \hat{\epsilon}, \quad G=\alpha \hat{G}, \\
F^{[v]}=K_{o}, \quad K_{c}=K_{o}, \quad R^{2}=\alpha \beta I, \quad \gamma=\gamma_{o}
\end{gathered}
$$

were $\alpha>0$ and $\beta>0$ are sufficiently small positive scalars.

(b) If the matrix inequalities (16), (17), (18), (19) are feasible for all vertices $v=1 \ldots \bar{v}$ where $S, R^{2}=$ $\operatorname{diag}\left(r^{2}\right), G, K_{c}=\operatorname{diag}\left(k_{c}\right), \gamma \epsilon, \alpha$ are decision variables common to all inequalities, and $P^{[v]}=P^{[v] T}$, $Y^{[v]}, F^{[v]}=\operatorname{diag}\left(f^{[v]}\right)$ are vertex-dependent decisions variables, then, whatever choice of $\Gamma=\operatorname{diag}(g) \succ$ 
$0, \Sigma=\operatorname{diag}(\sigma) \succeq 0$, the system (1), (4), (5) in closed-loop with the adaptive control (12), (14), (15) is such that

$$
\dot{V}(x, K, \xi)+2 \epsilon x^{T} E_{2}^{T} E_{2} x+\alpha\left(z^{T} z-\gamma^{2} w^{T} w\right)<8 \sum_{i=1}^{p} \sigma_{i} \frac{r_{i}{ }^{2}}{g_{i}}
$$

where $V(x, K, \xi)$ is a parameter-dependent Lyapunov function defined by

$$
V(x, K, \xi)=x^{T} E_{2}^{T} P(\xi) E_{2} x+\operatorname{Tr}\left[(K-F(\xi))^{T} \Gamma^{-1}(K-F(\xi))\right]
$$

with $P(\xi)=\sum_{v=1}^{\bar{v}} \xi_{v} P^{[v]}$ and $F(\xi)=\sum_{v=1}^{\bar{v}} \xi_{v} F^{[v]}$.

The proof is given in Appendix B.

The result of this theorem is an extension of the preliminary result in [23]. The novelties compared to that previous result are: $L_{2}$ type performance with respect to the pair of outputs-inputs $z / w$; Adaptive gains evolve in sets which are not centered at the initially chosen value $K_{o}$ but at a new value $K_{c}$ that should be designed; The Lyapunov function (21) is parameter-dependent not only for the quadratic in state $x$ term but also for the quadratic in state $K$ term. The improvement brought by $K_{c} \neq K_{o}$ provides new degrees of freedom that may lead to controllers with better closed-loop performance. It is indeed the case as illustrated on a numerical example in the next section. The improvement with respect to the parameter-dependent Lyapunov function reduces the conservatism of the results.

\subsection{Properties of the adaptive control law}

We shall now discuss the implications of (20) for the closed-loop system. This property will be named 'practical $L_{2}$ performance' meaning that it is almost the same as (8) which proves the $L_{2}$ performance of the LTI system. The difference is the right-hand side term than may be considered as small, which allows to conclude about practical stability only (convergence to a neighborhood of the equilibrium).

Consider for a start several critical cases.

- Assume that $\bar{r}=\max _{i=1}^{p} r_{i}=0$. This case corresponds to the situation without adaptation $(k(t)=$ $\left.k_{o}=f\right)$, the inequalities (16), (17), (18), (19) boil down to those of Assumption 3 and hence the smallest value of $\gamma$ solution of the matrix inequality constraints of Theorem 1 is exactly $\gamma_{o}$. In this case there is no adaptation, the system is trivially identical to the one with LTI control, and has the same performances.

- Assume that $\bar{r}>0$, there is hence room for adaptation, at least of one of the $p$ control parameters. The optimal value of $\gamma$ under constraints in Theorem 1 is, because of property (a), less or equal to the one obtained for the non-adaptive control: $\gamma \leq \gamma_{o}$. Because the conditions are not convex, there is no systematic method to find the adaptive control parameters such that $\gamma<\gamma_{o}$, but from a theoretical point of view it is attainable. We shall see on the numerical example that it is achievable in practice.

- Assume that $\bar{r}>0$ and $\bar{\sigma}=\max _{i=1}^{p} \sigma_{i}=0$, i.e. the adaptive rules are without damping terms. The adaptive gains are not required to converge asymptotically to the original non-adaptive values $k_{o}$. In this case equations (20) and (8) coincide. The same conclusions can be drawn, meaning that the adaptive and non adaptive control laws stabilize asymptotically the state $x$ to $E_{2} x=0$ when there are no perturbations. Moreover, for zero initial conditions $E_{2} x(0)=0$ one gets the following $L_{2}$ performance with adaptive control: $\|z\| \leq \gamma\|w\|$. Since it is possible to find solutions such that $\gamma \leq \gamma_{o}$, adaptive control can be proved to perform better than the initially provided LTI gain, in the sense that one gets a smaller guaranteed upper bound on the robust $L_{2}$ performance.

- Assume that $\bar{r}>0$ and $g=\min _{i=1}^{p} g_{i} \rightarrow \infty$, that is if one choses very high adaptation gains. Then the same conclusions as for the case $\bar{\sigma}=0$ apply asymptotically. This property shall not be used in practice because of implementation issues when choosing very high gains in the adaptation rule.

We now study the case when $\bar{r}>0, \bar{\sigma}>0, g<\infty$ and evaluate the consequences in terms of convergence of the state to a neighborhood of $E_{2} x=0$ (practical stability) and input/output performance during transients. The system is driven by the equations (1), (5), $u=K y,(14),(15)$ which may be summarized as

$$
\phi(\dot{x}, x, \dot{k}, k, w, \xi)=0, \quad z=\psi(x, w), \quad \underline{k} \leq k \leq \bar{k}
$$


where the state of the global non-linear system is composed of $x \in \mathbb{R}^{n_{x}}$ and $k \in \mathbb{R}^{p}, w \in \mathbb{R}^{m_{w}}$ is a perturbation input and $z \in \mathbb{R}^{p_{z}}$ is an output. For the closed-loop system we have built a quadratic Lyapunov like function which may be rewritten as

$$
V(x, k, \xi)=x^{T} E_{2}^{T} P(\xi) E_{2} x+(k-f(\xi))^{T} \Gamma^{-1}(k-f(\xi))
$$

and whose derivative satisfies

$$
\dot{V}(x, k, \xi)+2 \epsilon x^{T} E_{2}^{T} E_{2} x+\alpha\left(z^{T} z-\gamma^{2} w^{T} w\right)<8 p \bar{\sigma} \frac{\bar{r}^{2}}{\underline{g}} .
$$

We shall show the implications of this property in terms of practical stability. Assume that at all times $\|w(t)\| \leq \bar{w}$ where $\bar{w}$ is a bound on the euclidian norm of the instantaneous value of the perturbation. Then (24) implies that for all $t \geq 0$ :

$$
\dot{V}(x(t), k(t), \xi)+2 \epsilon x^{T}(t) E_{2}^{T} E_{2} x(t)<8 p \bar{\sigma} \frac{\bar{r}^{2}}{\underline{g}}+\alpha \gamma^{2} \bar{w}^{2} .
$$

Since $k_{i}$ and $f_{i}(\xi)$ are bounded in intervals centered at $k_{i c}$ of radius $r_{i}$ one gets that

$$
(k-f(\xi))^{T} \Gamma^{-1}(k-f(\xi)) \leq 4 p \frac{\bar{r}^{2}}{\underline{g}} .
$$

This bound is used to define the following scalar

$$
l_{1}(\xi)=\frac{\lambda_{\max }(P(\xi))}{2 \epsilon}\left(8 p \bar{\sigma} \frac{\bar{r}^{2}}{\underline{g}}+\alpha \gamma^{2} \bar{w}^{2}\right)+4 p \frac{\bar{r}^{2}}{\underline{g}}
$$

which is such that $V(x, k, \xi) \geq l_{1}(\xi)$ implies $2 \epsilon x^{T}(t) E_{2}^{T} E_{2} x(t)>8 p \bar{\sigma} \frac{\bar{r}^{2}}{\underline{g}}+\alpha \gamma^{2} \bar{w}^{2}$. We hence can conclude that all states satisfying $V(x, k, \xi) \geq l_{1}(\xi)$ are such that $\dot{V}(x, k, \xi)<0$. Lyapunov theory allows to conclude that the set $V(x, k, \xi) \leq l_{1}(\xi)$ is invariant and all trajectories starting out of this set converge asymptotically to it. Define the second scalar

$$
l_{2}^{2}=\max _{\xi \in \Xi_{\bar{v}}} \frac{l_{1}(\xi)}{\lambda_{\min }(P(\xi))}
$$

This value is such that $V(x, k, \xi) \leq l_{1}(\xi)$ implies $x^{T} E_{2}^{T} E_{2} x \leq l_{2}{ }^{2}$. We hence conclude that the norm of the vector $E_{2} x$ is robustly asymptotically less than $l_{2}$. The state $x$ converges to this neighborhood of the equilibrium subspace $E_{2} x=0$. It is a practical stability property in comparison to asymptotic convergence to the subspace itself.

Now assume that $x(0)=0, k(0)=k_{c}$ and define $\|z\|_{T}^{2}=\int_{0}^{T} z(t)^{T} z(t) d t$. To study the practical inputoutput performance of the system, take the integral from $t=0$ to $t=T$ of (24). It gives

$$
V(x(T), k(T), \xi)-\left(k_{c}-f(\xi)\right)^{T} \Gamma^{-1}\left(k_{c}-f(\xi)\right)+\alpha\left(\|z\|_{T}^{2}-\gamma\|w\|_{T}^{2}\right)<8 p T \bar{\sigma} \frac{\bar{r}^{2}}{\underline{g}}
$$

where $V(x(T), k(T), \xi)>0$. Moreover, since the elements of $f(\xi)$ are in intervals centered at $k_{c}$ with radius at most $\bar{r}$ one gets

$$
\alpha\left(\|z\|_{T}^{2}-\gamma\|w\|_{T}^{2}\right)<(8 p T \bar{\sigma}+1) \frac{\bar{r}^{2}}{\underline{g}}
$$

which also reads as

$$
\|z\|_{T}^{2}<\gamma\|w\|_{T}^{2}+\frac{8 p T \bar{\sigma}+1}{\alpha} \frac{\bar{r}^{2}}{\underline{g}}
$$

illustrating an upper bound on how much the 'practical $L_{2}$-performance' is a degraded version of the $L_{2}$ performance. For $\bar{\sigma} \neq 0$ the upper bounds on the additional terms are linear in time $T$. 


\subsection{Heuristic algorithm for adaptive control design}

The matrix inequality (19) is not linear and is not convex in the $K_{c}$ parameters. We therefore cannot expect a simple convex optimization result for the design of the $G, K_{c}$ and $R^{2}$ parameters that define the adaptive law. An iterative-LMI heuristic algorithm is proposed instead.

Let $\mathcal{M}\left(P^{[v]}, Y^{[v]}, F^{[v]}, S, G, K_{c}, R^{2}, \epsilon, \alpha, \gamma\right)$ be the matrix inequalities composed of (16), (17), (18), (19). Moreover let $\mathcal{M}_{1}\left(P^{[v]}, Y^{[v]}, F^{[v]}, S, G, K_{c}, \Delta_{c}, R^{2}, \epsilon, \alpha, \gamma\right)$ be the following matrix inequalities

$$
\begin{aligned}
& {\left[\begin{array}{cc}
R^{2} & K_{c}+\Delta_{c}-F^{[v]} \\
K_{c}+\Delta_{c}-F^{[v]} & I
\end{array}\right] \succeq 0, \quad\left[\begin{array}{cc}
R^{2} & K_{c}+\Delta_{c}-K_{o} \\
K_{c}+\Delta_{c}-K_{o} & I
\end{array}\right] \succeq 0,} \\
& \left(E_{2} E_{2}^{\circ}\right)^{T} P^{[v]}\left(E_{2} E_{2}^{\circ}\right) \succ 0, \\
& \left\{N_{2 x}^{T} P_{e}^{[v]} N_{1 x}+S M_{c}^{[v]}\right\}^{\mathcal{S}}+\epsilon N_{2 x}^{T} E_{2}^{T} E_{2} N_{2 x}+\alpha\left(N_{z}^{T} N_{z}-\gamma^{2} N_{w}^{T} N_{w}\right) \\
& +2 N_{y}^{T} R^{2} N_{y}-2 N_{y u}^{T} N_{K}^{T} N_{K} N_{y u}+\left\{-2 N_{y u}^{T}\left[\begin{array}{c}
\Delta_{c} \\
0
\end{array}\right] N_{K} N_{y u}+N_{y}^{T} G^{T} N_{F}^{[v]} N_{y u}\right\}^{\mathcal{S}} \prec 0 .
\end{aligned}
$$

The two problems are very similar. The second one amounts to replacing $K_{c}$ by an incremented version $K_{c}+\Delta_{c}$ and removing the second order term $\Delta_{c}{ }^{2}$. The algorithm that follows is build based on the following ideas:

- The starting point is the LTI controller $K_{o}$. Thanks to property (a) in Theorem 1 , we know that the constrains $\mathcal{M}$ shall be feasible under Assumption 3. Constraints $\mathcal{M}_{1}$ shall be feasible as well since $\mathcal{M}$ and $\mathcal{M}_{1}$ coincide for the choice of $\Delta_{c}=0$. Starting from this initialization value, the algorithm shall start by searching for an adaptive law with gains evolving in sets centered at this value $K_{o}$ and which will not degrade too much the performance. The requirement on the performance is set to be $\gamma_{1}=\delta \gamma_{o}$ where $\gamma_{o}$ is the solution to constraints in Assumption 3 and $\delta>1$ is the level of degradation. The larger is the scalar $\delta$ the more freedom is given to the initialization phase.

- For fixed values of $F^{[v]}, K_{c}$ and $\gamma$, the constraints $\mathcal{M}_{1}$ are linear in all other decision variables thus allowing the search for an increment $\Delta_{c}$. This increment is searched while trying to increase the intervals in which evolve the adaptive gains. More room for adaptation is expected to be profitable to the system. The objective to be optimized is a weighted sum of the elements in the diagonal matrix defining the size of the intervals: $\lambda^{T} R^{2} \lambda$ where $\lambda \in \mathbb{R}^{p}$ is a given vector.

The rationale for the choice of the parameter $\lambda$ is as described in the following example. Assume that $K_{o}=\operatorname{diag}\left(\left[\begin{array}{ll}1 & 10\end{array}\right]\right)$ is the initially given LTI control gain. One can a priori decide to search for intervals of adaptation that would be 10 times larger for the second coefficient compared to the first one. In percentage, compared to the initial value, the intervals would hence be similar. One way to do so, is to choose $\lambda=\left(\begin{array}{ll}1 & 10\end{array}\right)^{T}$ and hence to maximize $\lambda^{T} R^{2} \lambda=R_{1}^{2}+100 R_{2}^{2}$. The size of the adaptation interval for the second coefficient (given by $R_{2}$ ) having a stronger weight in the maximization will be larger, if the constraints allow it.

- The constraints $\mathcal{M}_{1}$ are first order approximations of the true constraints. $\Delta_{c}$ is hence to be understood as a gradient and a new value of $K_{c}$ may be searched for in this direction under constraints $\mathcal{M}$. The problem is not LMI but may be solved with a bisection over a positive scalar.

- For fixed values of $G, K_{c}, R^{2}$ and $\alpha$, the constraints $\mathcal{M}$ are linear and one may optimize over the $L_{2}$ performance indicator $\gamma$. This optimization can be understood as the analysis of the closed-loop for a given adaptive control law. The result is a new guess of Lyapunov function parameters $F^{[v]}$.

Based on these considerations the algorithm is as follows. It alternates LMI optimization steps where some of the Lyapunov decisions variables are fixed, with steps in which the control parameters are frozen. This strategy is rather classical for static output feedback design as reported in [31]. The interesting difference is that here the Lyapunov matrix $P(\xi)$ is optimized at all steps.

Algorithm 1 The algorithm assumes as inputs, a baseline LTI control gain $K_{o}$, a scalar $\delta>1$ and a vector $\lambda \in \mathbb{R}^{p}$. 


\section{- Initialization}

Compute the $L_{2}$ performance $\gamma_{o}$ using conditions of Assumption 3. The decision variables are $\hat{P}^{[v]}$, $\hat{Y}^{[v]}, \hat{S}, \hat{\epsilon}, \gamma_{o}^{2}$. If the conditions are infeasible, stop, the algorithm cannot be applied. Else, assign the following values: $\epsilon_{0}=\hat{\epsilon}, \gamma_{1}=\delta \gamma_{o}, F_{1}^{[v]}=K_{c, 1}=K_{o}, k=1$.

- Step $k, 1$

Maximize $\lambda^{T} R^{2} \lambda$ under constraints $\mathcal{M}_{1}\left(P^{[v]}, Y^{[v]}, F_{k}^{[v]}, S, G, K_{c, k}, \Delta_{c}, R^{2}, \epsilon_{0}, \alpha, \gamma_{k}\right)$. The decision variables are $P^{[v]}, Y^{[v]}, S, G, \Delta_{c}, R^{2}, \alpha$. At the optimum assign the following value $\Delta_{c, k}=\Delta_{c}$.

- Step $k, 2$

Search by bisection a maximal scalar $\beta \geq 0$ such that the following optimization problem is feasible:

$\operatorname{Max} \lambda^{T} R^{2} \lambda$ under $\mathcal{M}\left(P^{[v]}, Y^{[v]}, F_{k}^{[v]}, S, G, K_{c, k}+\beta \Delta_{c, k}, R^{2}, \epsilon_{0}, \alpha, \gamma_{k}\right)$.

The decision variables are $P^{[v]}, Y^{[v]}, S, G, \beta, R^{2}, \alpha$. At the optimum assign the following values $K_{c, k+1}=K_{c, k}+\beta \Delta_{c, k}, G_{k}=G, R_{k}^{2}=R^{2}, \alpha_{k}=\alpha$.

- Step $k, 3$

Minimize $\gamma$ under constraints $\mathcal{M}\left(P^{[v]}, Y^{[v]}, F^{[v]}, S, G_{k}, K_{c, k+1}, R_{k}^{2}, \epsilon_{0}, \alpha_{k}, \gamma\right)$.

The decision variables are $P^{[v]}, Y^{[v]}, F^{[v]}, S, \gamma$. At the optimum assign the following values $F_{k+1}^{[v]}=$ $F^{[v]}, \gamma_{k+1}=\gamma$.

\section{- Termination}

If $\gamma_{k}-\gamma_{k+1}$ is below some predefined threshold, stop, and assign $\bar{k}=k$. Else update $k \leftarrow k+1$ and go to Step $k, 1$.

Because of the iterative search with alternating decision variables, the algorithm is such that the sequence $\gamma_{k \geq 1}$ is decreasing: i.e. $0 \leq \gamma_{k+1} \leq \gamma_{k} \leq \delta \gamma_{o}$ for all $k \geq 1$. The algorithm is hence guaranteed to converge, but there is no expected property for the final value, except that the heuristic progresses slowly at that point. There is no guarantee either that it will converge to a value strictly less than the performance of the initial LTI control. $\gamma_{\bar{k}}<\gamma_{o}$ may not hold because $\delta>1$.

Initialization, steps $k, 1$ and $k, 3$ are LMI problems. Step $k, 2$ implies to solve several LMI problems for each step of the bisection. In our code we have chosen to search for $0 \leq \beta \leq 1$ and started the bisection with $\beta=1$. In almost all cases this choice proved to be feasible. Hence, in practice, the average of LMIs solved at Step $k, 2$ is close to one.

\section{Numerical example}

We shall as in [11] consider the quarter-car suspension system composed of a chassis with vertical position $h_{c}$ and the wheel with vertical position $h_{w}$. The dynamics of the system are given by

$$
m_{c} \ddot{h}_{c}=\pi \quad, \quad m_{w} \ddot{h}_{w}=-\pi+k_{w}\left(w-h_{w}\right)
$$

where $m_{c}$ is the mass of the chassis, $m_{w}$ the mass of the wheel, $k_{w}$ the wheel stiffness, $w$ is the height of the terrain relative to a mean position and $\pi$ is the force of interaction between chassis and the wheel. The link between the chassis and the wheel is composed of a spring with stiffness $k_{c}$, a damper of constant $c$ and an actuator $\tilde{u}$ :

$$
\pi=k_{c}\left(h_{w}-h_{c}\right)+c\left(\dot{h}_{w}-\dot{h}_{c}\right)+\tilde{u} .
$$

For this system we are interested in having a good performance in terms of the chassis position $z=h_{c}$ in response to disturbances $w$. The measurements are supposed to be limited to the chassis-wheel inter-distance $\tilde{y}=h_{c}-h_{w}$. Given these equations the plant is naturally in the following descriptor form

$$
\begin{aligned}
& {\left[\begin{array}{cccc}
m_{c} & 0 & 0 & 0 \\
0 & 1 & 0 & 0 \\
0 & 0 & m_{w} & 0 \\
0 & 0 & 0 & 1 \\
0 & 0 & 0 & 0
\end{array}\right] \dot{\tilde{x}}+\left[\begin{array}{c}
-1 \\
0 \\
1 \\
0 \\
1
\end{array}\right] \pi=\left[\begin{array}{cccc}
0 & 0 & 0 & 0 \\
1 & 0 & 0 & 0 \\
0 & 0 & 0 & -k_{w} \\
0 & 0 & 1 & 0 \\
-c & -k_{c} & c & k_{c}
\end{array}\right] \tilde{x}+\left[\begin{array}{c}
0 \\
0 \\
k_{w} \\
0 \\
0
\end{array}\right] w+\left[\begin{array}{l}
0 \\
0 \\
0 \\
0 \\
1
\end{array}\right] \tilde{u}} \\
& z=\left[\begin{array}{llll}
0 & 1 & 0 & 0
\end{array}\right] \tilde{x} \\
& \tilde{y}=\left[\begin{array}{llll}
0 & 1 & 0 & -1
\end{array}\right] \tilde{x}
\end{aligned}
$$


This simple example illustrates some of the advantages of our descriptor modeling framework. The $E_{x}$ matrix is affine in the parameters which would not be the case if working with non-descriptor state-space representations that would inevitably include inverses of the masses. Compared to the model that may be obtained when replacing $\pi$ by its expression as a function of the state $x$ : The interaction $\pi$ between the chassis and the wheel appear explicitly in the model which can be useful for understanding the structure of the model; The matrices are sparser which happens to be useful with respect to numerical issues. Compared to descriptor models where $\pi$ is included in the 'state' $x$, our model highlights which components are involved in the definition of stability and which are auxiliary for this issue.

We shall control this suspension with a second order control defined by

$$
\begin{aligned}
& \dot{\eta}=\left[\begin{array}{cc}
0 & 1 \\
-1+k_{1} & -2+k_{2}
\end{array}\right] \eta+\left[\begin{array}{l}
0 \\
1
\end{array}\right] \tilde{y} \\
& \tilde{u}=\left[\begin{array}{ll}
k_{3} & k_{4}
\end{array}\right] \eta+k_{5} \tilde{y}
\end{aligned}
$$

which may be rewritten as

$$
\begin{aligned}
& \dot{\eta}=\left[\begin{array}{cc}
0 & 1 \\
-1 & -2
\end{array}\right] \eta+\left[\begin{array}{lllll}
0 & 0 & 0 & 0 & 0 \\
1 & 1 & 0 & 0 & 0
\end{array}\right] u+\left[\begin{array}{l}
0 \\
1
\end{array}\right] \tilde{y} \\
& y=\left[\begin{array}{ll}
1 & 0 \\
0 & 1 \\
1 & 0 \\
0 & 1 \\
0 & 0
\end{array}\right] \eta+\left[\begin{array}{l}
0 \\
0 \\
0 \\
0 \\
1
\end{array}\right] \tilde{y} \\
& 0
\end{aligned}
$$

Applying results of Appendix A leads us to study the diagonal adaptive control of a linear descriptor system of order $n_{x}=5$.

The uncertain parameters are $m_{c} \in[320,384], m_{w} \in[38,42], k_{c} \in[171,189], k_{w} \in[180,220]$ and $c \in[950,1050]$. This generates polytopes of $\bar{v}=2^{5}=32$ vertices. The number of matrix inequalities and of the $P^{[v]}$ and $F^{[v]}$ variables is proportional to this value.

The Algorithm is applied with $K_{o}=0$ because the system is open-loop stable. The initialization step provides an upper bound on the $L_{2}$ performance of the open-loop system $\gamma_{o}=5.74153$. The parameter $\delta$ is

\begin{tabular}{|c|c|c|c|c|c|c|c|}
\hline$k$ & \multicolumn{3}{|c|}{$k_{c, k+1}$} & \multicolumn{3}{|c|}{$r_{k}$} & $\gamma_{k+1}$ \\
\hline 1 & -0.0003 & -0.0000 & 0.0005 & 2.7582 & 2.7582 & 14.5504 & 5.8833 \\
\hline 2 & -0.4662 & -2.2413 & -11.7436 & 4.8025 & 5.7323 & 29.3170 & 5.8021 \\
\hline 3 & -0.9452 & -3.5799 & -13.8372 & 4.6937 & 7.4833 & 32.2886 & 5.7396 \\
\hline 4 & -1.1673 & -3.8574 & -14.9313 & 3.6247 & 7.8346 & 33.8038 & 5.7115 \\
\hline 5 & -1.7560 & -4.4477 & -16.1724 & 3.4283 & 8.4603 & 35.0475 & 5.6941 \\
\hline 6 & -2.5678 & -5.1463 & -18.4074 & 3.7472 & 9.1833 & 37.3132 & 5.6931 \\
\hline 7 & -3.2517 & -5.7769 & -19.9285 & 3.8004 & 9.8363 & 38.9728 & 5.6913 \\
\hline 8 & -3.2561 & -5.7812 & -19.9405 & 3.6755 & 9.8594 & 38.9862 & 5.6847 \\
\hline 9 & -3.2625 & -5.7895 & -19.9662 & 3.4875 & 9.8810 & 39.0128 & 5.6840 \\
\hline
\end{tabular}
set to 1.05 and the weight to $\lambda=\left(\begin{array}{lll}0.1 & 0.1 & 1\end{array}\right)^{T}$. The iterations are summarized in Table 1 where $k_{c, k+1}$ is the row vector of the diagonal elements of $K_{c, k+1}$ and $r_{k}$ is the row vector of the diagonal elements of $R_{k}$.

Table 1: Iterations of the Algorithm for $K_{o}=0$

The algorithm was run on a MacBook Pro $2.9 \mathrm{GHz}$ Intel Core i5 with Matlab2016b. LMIs were coded using YALMIP (R20141030 release) by [24] and solved using SDPT3 (version 4.0) by [33]. The global solver time is about 3 minutes. The size of the LMI problem at initialization step is composed of 553 decision variables and has 544 rows. The matrix inequalities solved during the other steps have 980 decision variables and 838 rows.

At the final iteration the gains driving the adaptation are defined by

$$
G=\left[\begin{array}{ccc}
551.3594 & 551.3594 & -624.3645 \\
12.7737 & 12.7772 & -30.8624 \\
21.6927 & 21.6929 & -107.8905
\end{array}\right]
$$


The two first columns are identical which is not surprising since the two first signals of the measurements $y$ are the same.

The improvement in terms of value fo $\gamma$ due to the adaptive control is not large but this may be due to the choice of $K_{o}$ or to drawbacks in the heuristic algorithm. To test further the method, we perform the same procedure but with $K_{o}=\operatorname{diag}\left(k_{c, 9}\right)=\operatorname{diag}\left[\begin{array}{lll}-3.2625 & -5.7895 & -19.9662\end{array}\right]$. That is, taking as baseline control the center of the adaptation sets issued from the previous optimization. This value has theoretically no specific property, but it happens to stabilize robustly the plant and the initialization step provides an upper bound on the $L_{2}$ performance of the open-loop system $\gamma_{o}=5.83996$. This value of static feedback gains gives a worse (robust upper bound) performance than when $K_{o}=0$.

Table 2: Iterations of the Algorithm for $K_{o}=\operatorname{diag}\left(k_{c, 9}\right)$ from Table 1

\begin{tabular}{|c|c|c|c|c|c|c|c|}
\hline$k$ & \multicolumn{3}{|c|}{$k_{c, k+1}$} & \multicolumn{3}{|c|}{$r_{k}$} & $\gamma_{k+1}$ \\
\hline 1 & -3.2625 & -5.7895 & -19.9662 & & $\begin{array}{lll}0 & 0 & 0\end{array}$ & & 5.9842 \\
\hline 2 & -6.9346 & -12.2304 & -65.0503 & 6.9683 & 8.7498 & 56.3361 & 5.5541 \\
\hline 3 & -10.8279 & -18.6620 & -120.3926 & 10.5657 & 16.2310 & 120.0982 & 3.1116 \\
\hline 4 & -19.6059 & -32.5009 & -189.0948 & 44.7203 & 47.5955 & 197.5315 & 1.0009 \\
\hline
\end{tabular}

The iterations are summarized in Table 2. This time the adaptive control is proved to outperform significantly all other controllers. The adaptive control is characterized by the following matrices

$$
\begin{aligned}
& K_{o}=\operatorname{diag}\left[\begin{array}{lll}
-3.2625 & -5.7895 & -19.9662
\end{array}\right] \text {, } \\
& K_{c}=\operatorname{diag}\left[\begin{array}{lll}
-19.6059 & -32.5009 & -189.0948
\end{array}\right] \text {, } \\
& R=\operatorname{diag}\left[\begin{array}{lll}
44.7203 & 47.5955 & 197.5315
\end{array}\right] \text {, } \\
& G=\left[\begin{array}{ccc}
997.1959 & 997.1959 & -98.4674 \\
-35.6224 & -35.6224 & 2.4433 \\
-2.4199 & -2.4199 & -395.3527
\end{array}\right] \text {. }
\end{aligned}
$$

The degrees of freedom brought by the new decision variable $K_{c}$ are the source of the improvement.

\section{Conclusions}

The paper exposes new results for the analysis and the design of simple direct adaptive control laws for uncertain systems. The main focus is on robustness both in terms of performance to external disturbances and with respect to parametric uncertainties. One novelty is that results apply to systems represented in descriptor form. Another novelty is that the adaptive gains are bounded in sets for which are not centered at an a priori value. Conservatism is reduced compared to earlier results by the use of new parameter-dependent Lyapunov functions. Results are illustrated on a numerical example. The adaptive control law is designed with low computation burden although the complexity of the uncertain system is non-trivial. Additional experiments including simulations of the closed-loop will be performed in the close future.

\section{References}

[1] A. Astolfi, D. Karagiannis, and R. Ortega. Nonlinear and Adaptive Control with Applications. Communications and Control Engineering. Springer London, 2008.

[2] K.J. Åström and B. Wittenmark. Adaptive Control. Addison-Wesley, 1989.

[3] I. Bar-Kana. Parallel feedforward and simplified adaptive control. Int. J. of Adaptive Control and Signal Processing, 1(2):95-109, 1987.

[4] I. Barkana, M.C.M. Teixeira, and L. Hsu. Mitigation of symmetry condition in positive realness for adaptive control. Automatica, 42(9):1611-1616, September 2006.

[5] R. Ben Yamin, I. Yaesh, and U. Shaked. Robust discrete-time simple adaptive model following with guaranteed $H_{\infty}$ performance. In IEEE Conf. Decision and Control, pages 3245-3250, Cancun, December 2008. 
[6] R. Ben Yamin, I. Yaesh, and U. Shaked. Robust discrete-time simple adaptive regulation. Systems 8 Control Letters, 59:787-791, 2010.

[7] S. Boyd, L. El Ghaoui, E. Feron, and V. Balakrishnan. Linear Matrix Inequalities in System and Control Theory. SIAM Studies in Applied Mathematics, Philadelphia, 1994.

[8] V. Campos, A.-T. Nguyen, and R.M. Palhares. LMI-based adaptive control for uncertain polytopic systems. In IEEE Conf. Decision and Control, Las Vegas, 2016.

[9] D. Coutinho, A. Trofino, and M. Fu. Guaranteed cost control of uncertain nonlinear systems via polynomial Lyapunov functions. IEEE Trans. Automatic Control, 47(9):1575-1580, 2002.

[10] J. Doyle, A. Packard, and K. Zhou. Review of LFTs, LMIs and $\mu$. In IEEE Conf. Decision and Control, pages 1227-1232, Brighton, December 1991.

[11] Y. Ebihara, D. Peaucelle, and D. Arzelier. S-Variable Approach to LMI-based Robust Control. Communications and Control Engineering. Springer, 2015.

[12] A.L. Fradkov. Adaptive stabilization of a linear dynamic plant. Autom. Remote Contr., 35(12):19601966, 1974.

[13] A.L. Fradkov. Shunt output feedback adaptive controllers for nonlinear plants. In IFAC World Congress, pages 5304-5309, San Fransisco, 1996.

[14] A.L. Fradkov. Passification of non-square linear systems and feedback Yakubovich-Kalman-Popov lemma. European J. of Control, 6:573-582, 2003.

[15] A.L. Fradkov, I.V. Miroshnik, and V.O. Nikiforov. Nonlinear and Adaptive Control of Complex Systems. Kluwer Academic Publishers, Dordrekht, 1999.

[16] N. Hovakimyan and C. Cao. $L_{1}$ adaptive control theory - Guaranteed robustness with fast adaptation. SIAM, 2010.

[17] A. Ilchmann and T. Reis, editors. Differential-Algebraic Equations Forum. Springer, 2013-2017.

[18] P. Ioannou and J. Sun. Robust Adaptive Control. Prentice Hall, Inc, 1996.

[19] Z. Iwai and I. Mizumoto. Realization of simple adaptive control by using parallel feedforward compensator. Int. J. Control, 59:1543-1565, 1994.

[20] H. Kaufman, I. Barkana, and K. Sobel. Direct adaptive control algorithms. Springer, New York, 1998. Second Edition.

[21] M. Krstic, I. Kanellakopoulos, and P. Kokotovic. Nonlinear and Adaptive Control Design. John Wiley and Sons, 1995.

[22] M. Kuipers and P. Ioannou. Robust adaptive multiple model controller design for an airbreathing hypersonic vehicle model. In AIAA Guidance, Navigation and Control Conference, Honolulu, 2008.

[23] H. Leduc, D. Peaucelle, and C. Pittet. Adaptive control LMI-based design for descriptor systems rational in the uncertainties. In IFAC Workshop on Adaptation and Learning in Control and Signal Processing, Eindhoven, June 2016.

[24] J. Löfberg. YALMIP, 2014.

[25] I. Masubuchi, T. Akiyama, and M. Saeki. Synthesis of output-feedback gain-scheduling controllers based on descriptor LPV system representation. In IEEE Conf. Decision and Control, pages 6115-6120, Maui, December 2003.

[26] P. Parks. Liapunov redesign of model reference adaptive control systems. IEEE Trans. on Automatic Control, 11(3):362-367, 1966.

[27] D. Peaucelle, A. Fradkov, and B. Andrievsky. Passification-based adaptive control of linear systems: Robustness issues. Int. J. of Adaptive Control and Signal Processing, 22(6):590-608, August 2008. doi: 10.1002/acs.1009. 
[28] D. Peaucelle and A.L. Fradkov. Robust adaptive $L_{2}$-gain control of polytopic MIMO LTI systems - LMI results. Systems $\&$ Control Letters, 57(11):881-887, 2008.

[29] C. Pittet, A.R. Luzi, D. Peaucelle, J.-M. Biannic, and J. Mignot. In flight results of adaptive attitude control law for a microsatellite. CEAS Space Journal, 7(2):291-302, December 2015.

[30] J.B. Pomet and L. Praly. Adaptive nonlinear regulation: Estimation from the Lyapunov equation. IEEE Transactions on Automatic Control, 37(6):729-740, 1992.

[31] M.S. Sadabadi and D. Peaucelle. From static output feedback to structured robust static output feedback: A survey. Annual Reviews in Control, 42:11-26, 2016.

[32] M.M. Seron, D.J. Hill, and A.L. Fradkov. Nonlinear adaptive control of feedback passive systems. Automatica, 31:1053-1060, 1995.

[33] T.C. Toh, M.J. Todd, and R.H. Tutuncu. SDPT3 - a MATLAB software package for semidefinite programming. Optimization Methods and Software, 11:545-581, 1999.

[34] A. Trofino. Robust stability and domain of attraction of uncertain nonlinear systems. In American Control Conference, pages 3707-3711, Chicago, Il, June 2000.

[35] I. Yaesh and U. Shaked. Simplified adaptive control via improved robust positive real conditions. Systems \& Control Letters, 55:1033-1040, 2006.

[36] V. A. Yakubovich. Minimization of quadratic functionals under the quadratic constraints and the necessity of a frequency condition in the quadratic criterion for absolute stability of nonlinear control systems. Soviet Math. Doklady, 14:593-597, 1973.

\section{A Proof that Assumption 2 is lossless}

This assumption is without loss of generality. Indeed, assume the actual plant is of the type

$$
\begin{aligned}
& \tilde{E}_{x}(\xi) \dot{\tilde{x}}+\tilde{E}_{\pi}(\xi) \pi=\tilde{A}(\xi) \tilde{x}+\tilde{B}_{w}(\xi) w+\tilde{B}_{u}(\xi) \tilde{u} \\
& z=\tilde{C}_{z} \tilde{x}+\tilde{D}_{z w} w \\
& \tilde{y}=\tilde{C}_{y} \tilde{x}
\end{aligned}
$$

and is controlled by a dynamic controller defined in state-space as

$$
\begin{aligned}
& \dot{\eta}=\tilde{A}_{K}\left(k_{o}\right) \eta+\tilde{B}_{K}\left(k_{o}\right) \tilde{y} \\
& \tilde{u}=\tilde{C}_{K}\left(k_{o}\right) \eta+\tilde{D}_{K}\left(k_{o}\right) \tilde{y}
\end{aligned}
$$

where the matrices are affine in the coefficients of a vector $k_{o}$ of important control parameters (could be all the non-zero coefficients of the controller). Using linear-fractional transform (LFT) results [10] this controller may be re-written as

$$
\begin{aligned}
\dot{\eta} & =A_{K} \eta+B_{1} u+B_{2} \tilde{y} \\
y & =C_{1} \eta \quad+D_{12} \tilde{y} \quad, \quad u=K_{o} y \\
\tilde{u} & =C_{2} \eta+D_{21} u+D_{22} \tilde{y}
\end{aligned}
$$

where $K_{o}=\operatorname{diag}\left(k_{o}\right)$ is a diagonal matrix containing the coefficients of the vector $k$. Then this closed-loop system is the same as the following augmented descriptor system

$$
\begin{aligned}
& E_{x}(\xi)\left(\begin{array}{c}
\dot{\tilde{x}} \\
\dot{\eta}
\end{array}\right)+E_{\pi}(\xi) \pi=A(\xi)\left(\begin{array}{c}
\tilde{x} \\
\eta
\end{array}\right)+B_{w}(\xi) w+B_{u}(\xi) u \\
& z=C_{z}\left(\begin{array}{c}
\tilde{x} \\
\eta
\end{array}\right)+D_{z w} w \\
& y=C_{y}\left(\begin{array}{l}
\tilde{x} \\
\eta
\end{array}\right)
\end{aligned}
$$

controlled by $u=K_{o} y$ and where

$$
E_{x}(\xi)=\left[\begin{array}{cc}
\tilde{E}_{x}(\xi) & 0 \\
0 & I
\end{array}\right], \quad E_{\pi}(\xi)=\left[\begin{array}{c}
\tilde{E}_{\pi}(\xi) \\
0
\end{array}\right],
$$




$$
\begin{gathered}
A(\xi)=\left[\begin{array}{cc}
\tilde{A}(\xi)+\tilde{B}_{u}(\xi) D_{22} \tilde{C}_{y} & \tilde{B}_{u}(\xi) C_{2} \\
B_{2} \tilde{C}_{y} & A_{K}
\end{array}\right], \\
B_{w}(\xi)=\left[\begin{array}{c}
\tilde{B}_{w}(\xi) \\
0
\end{array}\right], \quad B_{u}(\xi)=\left[\begin{array}{c}
\tilde{B}_{u}(\xi) D_{21} \\
B_{1}
\end{array}\right],
\end{gathered} \begin{aligned}
& C_{z}=\left[\begin{array}{cc}
C_{z} & 0
\end{array}\right], \\
& C_{y}=\left[\begin{array}{ll}
D_{12} \tilde{C}_{y} & C_{1}
\end{array}\right] .
\end{aligned}
$$

\section{B Proof of Theorem 1}

\section{B.1 Proof of Property (a) in Theorem 1}

Let us denote $N_{(7)}^{[v]}$ the matrix on the left hand side of the $\prec$ sign in (7) and choose any matrix $\hat{G}$. Assuming that the $\bar{v}$ inequalities $N_{(7)}^{[v]} \prec 0$ hold, then for a sufficiently small values $\alpha>0$ and $\beta>0$ the following inequalities hold as well

$$
N_{(7)}^{[v]} \prec-2 \beta \hat{N}_{y}^{T} \hat{N}_{y}-\frac{\alpha}{2}\left(\hat{S} B_{u}^{[v]}-\hat{N}_{y}^{T} \hat{G}^{T}\right)\left(B_{u}^{[v] T} \hat{S}^{T}-\hat{G} \hat{N}_{y}\right) .
$$

where $\hat{N}_{y}=\left[\begin{array}{lll}0 & C_{y} & 0\end{array}\right]$. Apply a Schur complement argument to get

$$
\left[\begin{array}{cc}
N_{(7)}^{[v]}+2 \beta \hat{N}_{y}^{T} \hat{N}_{y} & \hat{S} B_{u}^{[v]}-\hat{N}_{y}^{T} \hat{G}^{T} \\
B_{u}^{[v] T} \hat{S}^{T}-\hat{G} \hat{N}_{y} & -\frac{2}{\alpha} I
\end{array}\right] \prec 0
$$

When rearranging the terms and multiplying the entire inequality by $\alpha$ one gets exactly (19) with $P=\alpha \hat{P}$, $Y=\alpha \hat{Y}, S=\left[\begin{array}{c}\alpha \hat{S} \\ 0\end{array}\right], \epsilon=\alpha \hat{\epsilon}, \gamma=\gamma_{o}, G=\alpha \hat{G}, F^{[v]}=K_{o}, K_{c}=K_{o}$ and $R^{2}=\alpha \beta I$. If it holds for $\gamma=\gamma_{o}$ then by convexity it also holds for any value $\gamma \geq \gamma_{o}$.

\section{B.2 Proof of Property (b) in Theorem 1}

By convexity of matrix inequalities, the fact that (18) holds for all $v=1 \ldots \bar{v}$ implies that for all $\xi \in \Xi_{\bar{v}}$ one has $\left(E_{2} E_{2}^{\circ}\right)^{T} P(\xi)\left(E_{2} E_{2}^{\circ}\right) \succ 0$. Therefore the function $x^{T} E_{2}^{T} P(\xi) E_{2} x$ is strictly positive for all $x$ such that $E_{2} x \neq 0$. The function (21) is suitable to prove asymptotic convergence to the sub-space defined by $E_{2} x=0$.

Consider now (19). By convexity of matrix inequalities, the fact that it holds for all vertices $v=1 \ldots \bar{v}$ implies that for all uncertainties $\xi \in \Xi_{\bar{v}}$ one has

$$
\begin{aligned}
\left\{N_{2 x}^{T} P_{e}(\xi) N_{1 x}\right. & \left.+S M_{c}(\xi)\right\}^{\mathcal{S}}+\epsilon N_{2 x}^{T} E_{2}^{T} E_{2} N_{2 x}+\alpha\left(N_{z}^{T} N_{z}-\gamma_{o}^{2} N_{w}^{T} N_{w}\right) \\
+ & 2 N_{y}^{T} R^{2} N_{y}-2 N_{y u}^{T} N_{K}^{T} N_{K} N_{y u}+\left\{N_{y}^{T} G^{T} N_{F}(\xi) N_{y u}\right\}^{\mathcal{S}} \prec 0
\end{aligned}
$$

where $P_{e}(\xi)=\sum_{v=1}^{\bar{v}} \xi_{v} P_{e}^{[v]}, M_{c}(\xi)=\sum_{v=1}^{\bar{v}} \xi_{v} M_{c}^{[v]}$ and $N_{F}(\xi)=\sum_{v=1}^{\bar{v}} \xi_{v} N_{F}^{[v]}$. We shall now consider the following vector

$$
\mu=\left(\begin{array}{c}
E_{2 x x} \dot{x}+E_{2 x \pi} \pi \\
x \\
w \\
\left(K-K_{o}\right) y
\end{array}\right) .
$$

Along trajectories of the closed-loop system (1), (5) with $u=K y$, it is easy to notice that the following properties hold

$$
\begin{gathered}
P_{e}(\xi) N_{1 x} \mu=E_{2}^{T} P(\xi) E_{2} \dot{x}, \quad M_{c}(\xi) \mu=0, \quad N_{2 x} \mu=x, \\
N_{z} \mu=z, \quad N_{w} \mu=w, \quad N_{y} \mu=y, \\
N_{K} N_{y u} \mu=\left(K_{c}-K\right) y, \quad N_{F}(\xi) N_{y u} \mu=(F(\xi)-K) y .
\end{gathered}
$$

Congruence of $\mu$ on (26) hence implies

$$
\begin{aligned}
& 2 \dot{x}^{T} E_{2}^{T} P(\xi) E_{2} x+2 \epsilon x^{T} E_{2}^{T} E_{2} x+\alpha\left(z^{T} z-\gamma^{2} w^{T} w\right) \\
& +2 y^{T}\left(R^{2}-\left(K_{c}-K\right)^{T}\left(K_{c}-K\right)+G^{T}(F(\xi)-K)\right) y<0 .
\end{aligned}
$$


Thanks to the properties of (14) the term $R^{2}-\left(K_{c}-K\right)^{T}\left(K_{c}-K\right)$ is always positive. Using the fact that $y^{T} G^{T}(K-F(\xi)) y=\operatorname{Tr}\left(y^{T} G^{T}(K-F(\xi)) y\right)=\operatorname{Tr}\left(y y^{T} G^{T}(K-F(\xi))\right)$ and that the $K$ and $F(\xi)$ are diagonal we get

$$
\begin{aligned}
2 \dot{x}^{T} E_{2}^{T} P(\xi) E_{2} x+2 \epsilon x^{T} & E_{2}^{T} E_{2} x+\alpha\left(z^{T} z-\gamma^{2} w^{T} w\right) \\
& -2 \sum_{i=1}^{p}\left(G_{i} y\right) y_{i}\left(k_{i}-f_{i}(\xi)\right)<0
\end{aligned}
$$

where the $f_{i}(\xi)$ are the diagonal elements of $F(\xi)$. We shall now consider the derivative of the parameterdependent Lyapunov function:

$$
\begin{aligned}
\dot{V}(x, K, \xi) & =2 \dot{x}^{T} E_{2}^{T} P(\xi) E_{2} x+2 \operatorname{Tr}\left[\dot{K}^{T} \Gamma^{-1}(K-F(\xi))\right] \\
& =2 \dot{x}^{T} E_{2}^{T} P(\xi) E_{2} x+2 \sum_{i=1}^{p} \dot{k}_{i} g_{i}{ }^{-1}\left(k_{i}-f_{i}(\xi)\right) \\
& =2 \dot{x}^{T} E_{2}^{T} P(\xi) E_{2} x+2 \sum_{i=1}^{p}\left(-G_{i} y y_{i}-\frac{\sigma_{i}}{g_{i}}\left(k_{i}-k_{o i}\right)+g_{i}{ }^{-1} q_{i}\right)\left(k_{i}-f_{i}(\xi)\right)
\end{aligned}
$$

Combining this to $(27)$ one gets

$$
\dot{V}(x, K, \xi)+2 \epsilon x^{T} E_{2}^{T} E_{2} x+\alpha\left(z^{T} z-\gamma^{2} w^{T} w\right)<2 \sum_{i=1}^{p}\left(-\frac{\sigma_{i}}{g_{i}}\left(k_{i}-k_{o i}\right)+g_{i}{ }^{-1} q_{i}\right)\left(k_{i}-f_{i}(\xi)\right)
$$

Now let us consider (16). With the same convexity arguments as earlier it implies that for all $\xi \in \Xi_{\bar{v}}$ one has

$$
\left[\begin{array}{cc}
R^{2} & K_{c}-F(\xi) \\
K_{c}-F(\xi) & I
\end{array}\right] \succeq 0
$$

which implies after a Schur complement argument that $R^{2} \succeq\left(K_{c}-F(\xi)\right)\left(K_{c}-F(\xi)\right)$, that is $F(\xi) \in \mathcal{E}\left(K_{c}, R\right)$. Because of this fact one concludes that $q_{i}\left(k_{i}-f_{i}(\xi)\right) \leq 0$ (see definition of $q_{i}$ ). Combining this to (28) one gets

$$
\dot{V}(x, K, \xi)+2 \epsilon x^{T} E_{2}^{T} E_{2} x+\alpha\left(z^{T} z-\gamma^{2} w^{T} w\right)<-2 \sum_{i=1}^{p} \frac{\sigma_{i}}{g_{i}}\left(k_{i}-k_{o i}\right)\left(k_{i}-f_{i}(\xi)\right)
$$

Because of (17), with the same reasoning as upper, $K_{o} \in \mathcal{E}\left(K_{c}, R\right)$. Hence $k_{o i}, k_{i}$ and $f_{i}(\xi)$ are bounded in intervals centered at $k_{i c}$ of radius $r_{i}$, and we get $k_{i}-k_{o i} \leq 2 r_{i}$ and $k_{i}-f_{i}(\xi) \leq 2 r_{i}$ thus proving (20). 